\title{
Tool to Assess Likelihood of Fasting Glucose ImpairmenT (TAG-IT)
}

\author{
Richelle J. Koopman, MD, MS ${ }^{1}$ \\ Arch G. Mainous III, PbD ${ }^{2}$ \\ Charles J. Everett, $P b D^{2}$ \\ Rickey E. Carter, $\mathrm{PbD}^{3}$ \\ ${ }^{1}$ Department of Family and Community \\ Medicine, University of Missouri, Columbia \\ ${ }^{2}$ Department of Family Medicine, Medical \\ University of South Carolina, Charleston \\ ${ }^{3}$ Biostatistics, Bioinformatics, and Epidemi- \\ ology, Medical University of South Caro- \\ lina, Charleston \\ Conflicts of interest: none reported
}

\begin{abstract}
PURPOSE Fifty-four million people in the United States have impaired fasting glucose (IFG); if it is identified, they may benefit from prevention strategies that can minimize progression to diabetes, morbidity, and mortality. We created a tool to identify those likely to have undetected hyperglycemia.

METHODS We undertook a cross-sectional analysis of existing data from the National Health and Nutrition Examination Survey (NHANES) 1999-2004 of 4,045 US adults aged 20 to 64 years who did not have a disgnosis of diabetes who had a measured fasting plasma glucose. Using characteristics that are selfreported or measured without laboratory data, we developed a logistic regression model predicting IFG and undiagnosed diabetes. Based on this model, we created TAG-IT (the Tool to Assess likelihood of fasting Glucose ImpairmenT), validated it using NHANES III, examined race and ethnicity subsets, and compared it with body mass index (BMI) alone.
\end{abstract}

RESULTS Predictors in the final tool were age, sex, BMI, family history of diabetes, resting heart rate, and history of hypertension (or measured high blood pressure), which yielded an area under the curve (AUC) of 0.740 , significantly better than $\mathrm{BMI}$ alone $(\mathrm{AUC}=0.644)$.

CONCLUSIONS The TAG-IT efficiently identifies those most likely to have abnormal fasting glucose and can be used as a decision aid for screening in clinical and population settings, or as a prescreening tool to help identify potential participants for research. The TAG-IT represents an improvement over BMI alone or a list of risk factors in both its utility in younger adult populations and its ability to provide clinicians and researchers with a strategy to assess the risks of combinations of factors.

Ann Fam Med 2008;6:555-561. DOI: 10.1370/afm.913.

\section{INTRODUCTION}

$\mathrm{N}$ Tearly 54 million Americans, 26.0\% of US adults, have impaired fasting glucose (IFG). ${ }^{1}$ The incidence of progression to diabetes from IFG is nearly $2 \%$ per year, almost double the average incidence for diabetes. ${ }^{2}$ Additionally, IFG is associated with cardiovascular and other morbidities. ${ }^{3-5}$

Screening for asymptomatic IFG and undiagnosed diabetes could be considered, although population screening for diabetes using fasting plasma glucose levels is neither cost-effective nor recommended. . $^{6-8}$ Recently, however, there is increasing evidence that (1) prediabetes states are themselves associated with comorbidities and complications, and (2) strategies to prevent diabetes in those with prediabetes states are effective. ${ }^{4,5,9-17}$ It may therefore be beneficial to identify a group at increased risk for having prediabetes. Measuring fasting plasma glucose may be the best initial screening strategy for prediabetes, assuming low rates of oral glucose tolerance testing. ${ }^{9}$

The American Diabetes Association (ADA) lists risk factors that should trigger screening for prediabetes, which include an increased body mass 
index (BMI) plus 1 other risk factor, or age 45 years or older. ${ }^{18}$ The difficulty for clinicians lies in assessing the relative value of combinations of risk factors. For example, should a 22-year-old college athlete with a BMI of $26 \mathrm{~kg} / \mathrm{m}^{2}$ and a family history of diabetes be tested? Should a 35-year-old sedentary hypertensive woman be tested even though her BMI is $23 \mathrm{~kg} / \mathrm{m}^{2}$ ? A tool that could weigh the relative contributions of multiple risk factors would be helpful for clinicians over and above the ADA guidelines. ${ }^{18}$

One way to identify IFG may be to use risk scores that have been developed to detect undiagnosed diabetes. The Danish Diabetes Risk Score was developed in a Danish population aged 30 to 60 years as a selfadministered tool to identify undiagnosed diabetes. ${ }^{19}$ Similar risk scores have been developed for use in populations older than 40 years..$^{20,21}$ The average age of diagnosis for diabetes in the United States is 46 years. ${ }^{22}$ Given the increasing burden of type 2 diabetes in youth populations, however, we would expect IFG to occur at an even younger age. Accordingly, a tool that indicates the likelihood of undiagnosed diabetes and IFG in young adults is preferred.

We created a model and subsequent tool to assess the likelihood of fasting glucose impairment (TAG-IT, Tool to Assess likelihood of fasting Glucose ImpairmenT) with the aim of identifying people who have fasting hyperglycemia, comprising both IFG and undiagnosed diabetes. We then validated TAG-IT in a second population-based sample, and compared TAGIT with BMI alone for the ability to predict IFG and undiagnosed diabetes.

\section{METHODS}

\section{Data Source and Variables Examined}

We analyzed cross-sectional data from 4,045 participants, aged 20 to 64 years, from the population-based National Health and Nutrition Examination Survey (NHANES), 1999-2004. The NHANES participants are a stratified, multistage probability sample of the civilian, noninstitutionalized US population. The sampling design and weighting system of the survey allows estimates of the number and percentage of persons with selected diseases and risk factors, such as national estimates of the prevalence of diabetes, undiagnosed diabetes, and IFG. ${ }^{1}$ After applying appropriate sample weights, the unweighted sample of 4,045 participants represents more than 153 million Americans. We used SUDAAN software (RTI International, Research Triangle Park, North Carolina, 2005) to analyze this clustered probability sample and make population estimates. ${ }^{23}$ This study was approved by the Institutional Review Board of the Medical University of South Carolina.
Analysis was limited to participants with no history of diabetes who had a measured fasting plasma glucose. Participants with previously diagnosed diabetes were excluded based on the question, "Other than during pregnancy, have you ever been told by a doctor or health professional that you have diabetes or sugar diabetes?" Individuals were defined as having undiagnosed diabetes if they answered "no" to this question and had a fasting plasma glucose of 126 $\mathrm{mg} / \mathrm{dL}(7.0 \mathrm{mmol} / \mathrm{L})$ or greater.

We only examined predictors that could be selfreported or measured without obtaining a blood sample, because if a blood test was required, it would be much more sensible to measure fasting plasma glucose itself. ${ }^{24}$ Variables examined included age, sex, family history of diabetes in a first-degree relative, BMI, waist circumference, activity level, resting heart rate, history of hyperlipidemia, and history or presence of hypertension. A person was defined as having a history or presence of hypertension if they answered yes to the question, "Has a doctor or other health professional ever told you that you had hypertension, also called high blood pressure?" or if they had an average of 3 blood pressure measurements with a systolic pressure of $140 \mathrm{~mm} \mathrm{Hg}$ or higher or a diastolic pressure of 90 mm Hg or higher. Blood pressure was measured in the NHANES using procedures consistent with American Heart Association guidelines. ${ }^{25}$

Among the risk factors indicated by the ADA that should trigger consideration for screening for diabetes, ${ }^{18}$ we were not able to include a history of gestational diabetes, peripheral arterial disease, acanthosis nigricans, or polycystic ovary syndrome, as this information was not available to us. It could be argued, however, that these conditions should trigger an investigation of plasma glucose levels in and of themselves. Whereas ankle-brachial index, a good indicator of peripheral arterial disease, was measured in the NHANES for participants aged 40 years and older, it was not measured in younger participants, and it also did not fit our criterion of being a measurement that could be selfadministered or performed routinely in the setting of a health fair or primary care office visit.

We did not examine race or ethnicity specifically as a risk factor because we wanted to make a tool that would be generalizable. Rather, we created a model and the subsequent TAG-IT score from a populationbased sample with a race/ethnicity mix representative of the US population, and then we examined validity of the final risk score for specific race and ethnicity subsets as part of the validation analysis.

Our outcome was to predict abnormal fasting glucose, defined as fasting plasma glucose of $100 \mathrm{mg} / \mathrm{dL}$ or greater, which identifies those with IFG, as well as 
those with undiagnosed diabetes. We considered only examining those with IFG and not those with undiagnosed diabetes; in clinical practice, however, the tool will naturally identify those with undiagnosed diabetes, although proportionally this number will be small compared with those with IFG. ${ }^{1}$

\section{Creating the Model}

We used logistic regression in SUDAAN, with an initial forced inclusion model. In the initial model, we included age, sex, BMI, waist circumference, family history of diabetes, exercise history, hypertension, and history of hyperlipidemia to find the model that would best predict the abnormal fasting glucose logistic outcome. We examined hypertension as a predictor in different models as history of vs measured hypertension, including diastolic blood pressure, systolic blood pressure, a combination of systolic and diastolic blood pressure, and finally a combination of history of hypertension and elevated measured blood pressure. We separately examined resting heart rate in an additional model, a factor that differs between the TAG-IT score and the Danish Diabetes Risk Score. ${ }^{19}$ We stratified variables, eg, age, into groups to improve discrimination for the eventual score. We selected combinations of factors that were significant predictors in the initial model and examined multiple models to form the most parsimonious final model with the greatest predictive value.

\section{Converting to Integer Risk Points}

It is possible to use odds ratios from the regression model as risk points for the purposes of computing a total risk score, especially when a link to a computerized calculation of the score is generated. ${ }^{26}$ TAG-IT, however, is designed to be used in clinical as well as preclinical settings, so we preferred the ability to do a simple paper-and-pencil calculation of the score. For this reason, we rounded to whole number risk points, using the method of Charlson in developing the Charlson Comorbidity Index. ${ }^{27}$ In this scheme, significant odds ratios between 1 and 1.19 scored 0 points, ratios from 1.2 to 1.49 were rounded to 1 point, ratios from 1.5 to 2.49 were rounded to 2 points, and so forth.

\section{Validation and Comparison With Other Predictive Measures}

TAG-IT was validated using NHANES III, a population-based sample spanning the years 1988-1994. These NHANES data are not part of a cohort design; therefore, NHANES III (1998-1994) does not contain data from the same persons as the NHANES 1999-2004.

For validation using NHANES III, we again used data from adults aged 20 to 64 years. The TAG-IT scoring system was applied to each member of the
NHANES III validation sample. The outcome predicted by TAG-IT was compared with the true outcome (measure fasting plasma glucose), generating true-positive, true-negative, false-positive, and falsenegative outcome. These values were used to generate a receiver operating characteristic (ROC) curve. Finally, the area under the ROC curve (AUC) for the validation data set (NHANES III) was compared with the AUC for the development data set (NHANES 1999-2004) using MedCalc statistical software to test for a significant difference in the AUCs. ${ }^{28-30}$

We also examined the performance of TAG-IT within specific race and ethnic subsamples in NHANES 1999-2004. Race/ethnicity is self-defined by NHANES participants as "non-Hispanic white," "non-Hispanic black," "Hispanic," and "other." We validated the TAGIT score among these groups with the exception of the "other" group, which was too heterogeneous and small for specific examination.

An important consideration in the creation of the TAG-IT is whether the tool represents an improvement over other known strategies. We compared the ability of TAG-IT to predict IFG with BMI alone.

\section{RESULTS}

Among the 153 million adult participants aged 20 to 64 years who did not have known diabetes (unweighted $\mathrm{n}=4,045), 37.9$ million had a fasting plasma glucose level consistent with IFG (unweighted $n=1,014$ ), and 3.3 million had a fasting plasma glucose level consistent with previously undetected diabetes (unweighted $\mathrm{n}=103$ ). Table 1 displays the characteristics of the weighted NHANES 1999-2004 sample with respect to the predictor variables used in the initial full model.

History of high cholesterol and waist circumference were not significant in the initial model and were dropped from the final model. The odds ratios for the final model and risk point derivation are displayed in Table 2. Our preliminary investigation included participants aged 65 years and older. Because those aged 65 years and older scored 7 risk points in an initial model (odds ratio $[\mathrm{OR}]=7.03 ; 95 \%$ confidence interval [CI] 5.06-9.76), they were excluded from the sample used to derive the final model $l_{i}$ in essence, we showed that all those aged 65 years and older have a high likelihood of abnormal fasting glucose regardless of other characteristics. Accordingly, we limited analysis to data from those aged 20 to 64 years to best examine how to discriminate who among this younger group has a higher likelihood of abnormal fasting glucose. We excluded exercise history from the final model. Although a history of no regular exercise was significant in the model $(\mathrm{OR}=1.32 ; 95 \%$ 


\begin{tabular}{|c|c|c|}
\hline Characteristic & $\begin{array}{l}\text { Proportion } \\
\text { of Weighted } \\
\text { Sample }(\%)^{\text {a }}\end{array}$ & $\begin{array}{c}\text { Number } \\
\text { in Weighted } \\
\text { Sample } \\
\text { (Millions) }^{a}\end{array}$ \\
\hline \multicolumn{3}{|l|}{ Fasting glucose, mg/dL } \\
\hline$<100$ & 73.1 & 112.3 \\
\hline $100-125$ & 24.7 & 37.9 \\
\hline$\geq 126$ & 2.2 & 3.3 \\
\hline \multicolumn{3}{|l|}{ Age, years } \\
\hline $20-27$ & 18.4 & 28.2 \\
\hline $28-35$ & 20.0 & 30.8 \\
\hline $36-44$ & 23.1 & 35.4 \\
\hline $45-64$ & 38.5 & 59.1 \\
\hline \multicolumn{3}{|l|}{ Sex } \\
\hline Male & 48.9 & 75.1 \\
\hline Female & 51.1 & 78.4 \\
\hline \multicolumn{3}{|l|}{ Race/ethnicity } \\
\hline Non-Hispanic white & 71.9 & 110.4 \\
\hline Non-Hispanic black & 11.0 & 16.9 \\
\hline Hispanic & 13.1 & 20.2 \\
\hline Other & 3.9 & 6.0 \\
\hline \multicolumn{3}{|l|}{ Body mass index, $\mathrm{kg} / \mathrm{m}^{2}$} \\
\hline$<25$ & 36.9 & 56.7 \\
\hline $25-29.9$ & 34.1 & 52.4 \\
\hline$\geq 30$ & 29.0 & 44.4 \\
\hline \multicolumn{3}{|l|}{ Family history of diabetes } \\
\hline No & 50.5 & 77.5 \\
\hline Yes & 49.5 & 76.0 \\
\hline \multicolumn{3}{|l|}{ Heart rate, beats per min } \\
\hline$<60$ & 14.9 & 22.9 \\
\hline $60-69$ & 31.2 & 47.9 \\
\hline $70-79$ & 32.1 & 49.3 \\
\hline $80-89$ & 15.0 & 23.0 \\
\hline $90-99$ & 5.0 & 7.7 \\
\hline$\geq 100$ & 1.7 & 2.6 \\
\hline \multicolumn{3}{|l|}{ Hypertension } \\
\hline No & 72.8 & 111.8 \\
\hline Yes & 27.2 & 41.7 \\
\hline \multicolumn{3}{|l|}{ Waist circumference, $\mathrm{cm}$} \\
\hline Male $<102$, female $<88$ & 54.4 & 83.5 \\
\hline Male $\geq 102$, female $\geq 88$ & 45.6 & 70.0 \\
\hline \multicolumn{3}{|l|}{ Exercise } \\
\hline None & 34.6 & 53.2 \\
\hline Moderate & 27.3 & 41.9 \\
\hline Vigorous & 38.1 & 58.4 \\
\hline \multicolumn{3}{|l|}{ Hypercholesterolemia } \\
\hline No or not tested & 78.2 & 120.1 \\
\hline Yes & 21.8 & 33.5 \\
\hline
\end{tabular}

CI, 1.05-1.65), resting heart rate provided similar predictive ability in the model when substituted for exercise history; therefore, we chose resting heart rate for the model as it is easily measured and more easily extracted from an electronic health record.

Compared with women, men had an increased risk for IFG in the final model $(\mathrm{OR}=2.58 ; 95 \% \mathrm{CI}$, 2.24-2.98); being male conferred 3 risk points. In the NHANES sample, men had a higher mean fasting plasma glucose levels, $98.4 \mathrm{mg} / \mathrm{dL}$, than women, 93.5 $\mathrm{mg} / \mathrm{dL}(P<.001)$, consistent with the findings of previous examinations of the distribution of IFG among men and women in the United States. ${ }^{1}$

In Table 3 the AUC for TAG-IT is compared with the AUC for BMI alone in NHANES 1999-2004, as well as in the validation data set from NHANES III. Additionally, Table 3 displays the AUC for different race and ethnic subsets in the NHANES 1999-2004. TAG-IT provides greater predictive ability than BMI alone in the 1999-2004 US population, and in the 3 race and ethnic subsets. The ROC curves for the TAG-IT and BMI alone are depicted in Figure 1. Additionally, the AUC for the TAG-IT was significantly

\section{Table 2. Logistic Regression Model Predicting Fasting Glucose $\geq 100 \mathrm{mg} / \mathrm{dL}$ and Scores Assigned to Each Variable}

\begin{tabular}{|c|c|c|c|}
\hline Characteristic & OR & $95 \% \mathrm{Cl}$ & Score \\
\hline \multicolumn{4}{|l|}{ Age, years } \\
\hline $20-27$ & 1.00 & - & 0 \\
\hline $28-35$ & 1.47 & $1.08-2.00$ & 1 \\
\hline $36-44$ & 2.27 & $1.71-3.03$ & 2 \\
\hline $45-64$ & 4.36 & $3.38-5.64$ & 4 \\
\hline \multicolumn{4}{|l|}{ Sex } \\
\hline Male & 2.58 & $2.24-2.98$ & 3 \\
\hline Female & 1.00 & - & 0 \\
\hline \multicolumn{4}{|c|}{ Body mass index $\left(\mathrm{kg} / \mathrm{m}^{2}\right)$} \\
\hline$<25$ & 1.00 & - & 0 \\
\hline $25-29.9$ & 2.11 & $1.68-2.65$ & 2 \\
\hline$\geq 30$ & 2.82 & $2.24-3.55$ & 3 \\
\hline \multicolumn{4}{|c|}{ Family history of diabetes } \\
\hline No & 1.00 & - & 0 \\
\hline Yes & 1.27 & $1.07-1.52$ & 1 \\
\hline \multicolumn{4}{|c|}{ Heart rate (beats per min) } \\
\hline$<60$ & 1.12 & $0.85-1.47$ & 0 \\
\hline $60-69$ & 1.00 & - & 0 \\
\hline $70-79$ & 1.38 & $1.09-1.74$ & 1 \\
\hline $80-89$ & 1.56 & $1.18-2.05$ & 2 \\
\hline $90-99$ & 2.20 & $1.48-3.27$ & 2 \\
\hline$\geq 100$ & 4.02 & $1.97-8.19$ & 4 \\
\hline \multicolumn{4}{|l|}{ Hypertension } \\
\hline No & 1.00 & - & 0 \\
\hline Yes & 1.28 & $1.02-1.61$ & 1 \\
\hline
\end{tabular}


Table 3. Area Under the ROC Curve for TAG-IT Compared With BMI Alone for the Whole Sample and for Race/Ethnicity Subsamples (NHANES 1999-2004) and Validation Sample (NHANES 3)

\begin{tabular}{|c|c|c|}
\hline \multirow[b]{2}{*}{ Sample } & \multicolumn{2}{|c|}{ Area Under ROC Curve } \\
\hline & TAG-IT & BMI \\
\hline \multicolumn{3}{|l|}{ NHANES 1999-2004 } \\
\hline Whole sample & 0.740 & $0.644^{*}$ \\
\hline Non-Hispanic white & 0.753 & $0.669 *$ \\
\hline Non-Hispanic black & 0.717 & $0.656^{*}$ \\
\hline Hispanic & 0.724 & $0.564^{*}$ \\
\hline \multicolumn{3}{|l|}{ NHANES 3} \\
\hline Whole sample & 0.744 & $0.685^{*}$ \\
\hline
\end{tabular}

$\mathrm{BMI}=$ body mass index. NHANES = National Health and Nutrition Examination Survey; ROC $=$ receiver operating characteristic; TAG-IT $=$ Tool to Assess Likelihood of Fasting Glucose ImpairmenT.

*Significantly different from TAG-IT $(P \leq .05)$.

Figure 1. ROC curves for prediction using TAG-IT and BMI in the derivation sample (NHANES 1999-2004).

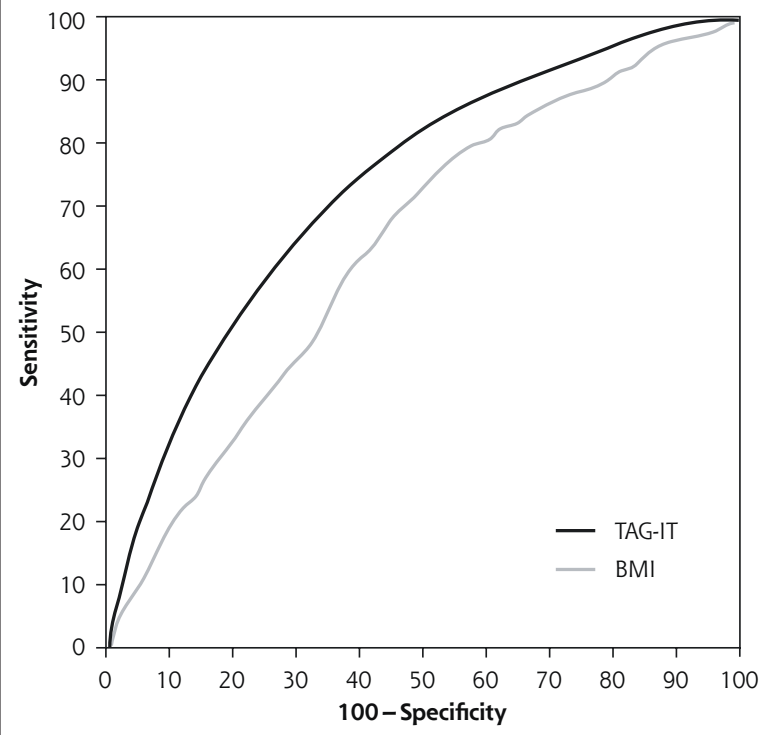

$\mathrm{BMI}=$ body mass index; $\mathrm{ROC}=$ receiver operating characteristic; $\mathrm{TAG}-\mathrm{IT}=$ Tool to Assess Likelihood of Fasting Glucose ImpairmenT.

Table 4. Sensitivity, Specificity, and Likelihood Ratios for Selected Risk Point Levels of TAG-IT

\begin{tabular}{lcccc}
\hline Criterion & $\begin{array}{c}\text { Sensitivity } \\
\mathbf{( \% )}\end{array}$ & $\begin{array}{c}\text { Specificity } \\
\mathbf{( \% )}\end{array}$ & $\begin{array}{c}\text { Positive } \\
\text { Likelihood } \\
\text { Ratio }\end{array}$ & $\begin{array}{c}\text { Negative } \\
\text { Likelihood } \\
\text { Ratio }\end{array}$ \\
\hline 5 or higher & 87.0 & 43.3 & 1.53 & 0.30 \\
6 or higher & 78.9 & 56.3 & 1.80 & 0.38 \\
7 or higher & 66.5 & 69.2 & 2.16 & 0.48 \\
8 or higher & 53.7 & 78.8 & 2.54 & 0.59 \\
9 or higher & 39.5 & 87.9 & 3.26 & 0.69 \\
\hline TAG-IT = Tool to Assess likelihood of fasting Glucose Impairment. \\
\hline
\end{tabular}

better than that of the Danish Diabetes Risk Score in the derivation sample, and equivalent in the validation sample (data not shown). ${ }^{19}$

The sensitivity and specificity at different scores for the TAG-IT, as well as the positive and negative likelihood ratios, are displayed in Table 4. Although a score of 5 or higher yields a high sensitivity, different cutoffs to enhance sensitivity, specificity, or positive likelihood ratio may be desired, depending on screening objectives.

\section{DISCUSSION}

We created TAG-IT, a tool to identify those at highest risk for having undetected hyperglycemia, comprising IFG and undiagnosed diabetes, with potential utility in both clinical and research settings. Whereas population screening for diabetes and perhaps IFG, by extension, has not been recommended in the past, ${ }^{6,7}$ there is growing evidence of the harms associated with prediabetes states and evidence that diabetes can be prevented. ${ }^{3,10-16}$ Additionally, communication with patients about disease risk has been shown to have a beneficial effect on therapeutic outcomes. ${ }^{31}$ A tool to identify efficiently those most likely to have an abnormal fasting glucose may affect our approach to decisions about screening and risk assessment.

We designed TAG-IT to use only those factors that are either self-reported or easily measured (eg, BMI). One consequence of this design and of our decision to exclude exercise history is that all the risk factors included in TAG-IT can be found in an electronic health record. By applying TAG-IT to patients in a practice panel by using its electronic health record, clinicians can potentially improve detection of IFG and, parenthetically, undetected diabetes. Additionally, this prescreening tool can be used at population-based screening events. It also has potential application as a Web-based screening tool to improve awareness and population health and to encourage compliance with physician-recommended lifestyle changes. ${ }^{31}$ Finally,

TAG-IT's emphasis on investigating the history and consequences of prediabetes states may help investigators to efficiently identify potential research participants

What score derived from the TAG-IT should trigger additional testing? This complex decision involves considering the goals of a screening program, the prevalence of disease in the target population, the strategy to be used for additional testing, and the cost of additional testing, all balanced against the perceived benefits of early detection. If high sensitivity is desired, with an aggressive case-finding strategy, then a 
score of 5 or higher $(87.0 \%$ sensitivity, Table 4$)$ might be chosen. On the other hand, if a high specificity is desired, minimizing additional testing and false-positive results, a score of 8 or $9(78.8 \%, 87.9 \%$ specificity, Table 4) might be used. An economic analysis would be the next step in making an informed decision about the threshold that should trigger additional testing.

Although TAG-IT has many advantages, we should note limitations in its design. First, this tool was developed from cross-sectional data and examines only present risk of elevated fasting plasma glucose. This score does not predict future risk for disease, as do published prospective risk scores for diabetes. ${ }^{32,33}$ Second, TAG-IT examines only fasting plasma glucose as an outcome. Although there is substantial overlap between IFG and impaired glucose tolerance, the TAG-IT does not address impaired glucose tolerance, which would require an oral glucose tolerance test. When considering probable nonadherence rates with the oral glucose tolerance test, however, using fasting plasma glucose levels may be the best initial strategy to screen for prediabetes. ${ }^{9}$

Even though diabetes and IFG are known to be more prevalent in some race and ethnic subgroups in the United States, ${ }^{1}$ we chose not to include race/ethnicity as a predictor in the TAG-IT. This decision was the result of careful consideration of precedent for exclusion of race/ethnicity in many other risk scores, ${ }_{1}^{19,21,34,35}$ and of the eventual application of TAG-IT in other countries and settings. Health and health research are increasingly global activities; creating a risk score that would only be applicable to the 3 most common race and ethnic subgroups in the United States seemed to unnecessarily limit the potential use of the tool.

Two advantages of TAG-IT are that it was developed from a population-based sample, increasing its validity to be widely applied, and that it is able to assess risk starting with adults aged 20 years. Indeed, the tool probably has greatest utility and discriminative ability among young adults. Given trends in diabetes age of diagnosis, and that IFG precedes onset of diabetes, the score's applicability to young adults is crucial. ${ }^{22}$

One main factor driving the development of TAGIT was the lack of guidance for clinicians about how to think about multiple risk factors, especially when some factors confer increased risk, while others confer decreased risk. TAG-IT goes beyond the list of risk factors proposed by the ADA ${ }^{18}$ providing additional guidance to clinicians faced by patients with combinations of risk factors. In the introduction, we proposed 2 examples, one a male college athlete, the other a sedentary woman with hypertension. If we presuppose that the athlete has a resting heart rate of 64 beats per minute and that the sedentary woman has a rest- ing heart rate of 85 beats per minute, we see that the athlete scores 3 points for being male, 2 for a BMI of $26 \mathrm{~kg} / \mathrm{m}^{2}$, and 1 for family history of diabetes, for a total of 6 points. The woman scores 1 point for her age of 35 years, 2 for a resting heart rate of 85 beats per minute, and 1 for hypertension, for a total of 4 points. In this case, the college athlete scores higher than his older sedentary counterpart, with a higher likelihood of IFG, mostly based on his higher BMI and male sex. This result might not be immediately intuitive without using TAG-IT.

The development of this tool raises questions about how it should be used. There may be harms associated with identifying IFG that affect a person's self-concept of health and well-being. Balanced against this potential harm is the benefit of intervening to decrease risk of development of diabetes and other complications. If we choose to use the tool, we need to determine the correct screening strategy. Ultimately, the question remains: What real value does earlier identification serve in terms of reductions in morbidity and mortality? Further investigations are necessary to address this question as well as the best screening strategies, including field testing for usability, qualitative investigations of the effects of a positive screening result on a person's concept of his or her health, and clinical trials assessing the effect of screening and communications on patient-oriented outcomes, such as weight loss, lifestyle change, and diabetes prevention.

In conclusion, TAG-IT represents an improvement over previous lists of risk factors and over BMI alone because it aids clinicians in combining multiple risk factors, it can be applied to young and middle-aged adults, it performs well in racial and ethnic subgroups, and it was developed from a population-based sample and validated in a second population-based sample. TAG-IT can be readily and immediately applied in clinical settings, can aid in the identification of potential research participants with IFG, can be widely applied in practices using electronic health records, and can improve the efficiency of population-based screening, including community and Web-based applications.

To read or post commentaries in response to this article, see it online at http://www.annfammed.org/cgi/content/full/6/6/555.

Key words: Hyperglycemia; impaired fasting glucose; risk assessment; diabetes mellitus; NHANES; nutrition surveys

Submitted October 30, 2007; submitted, revised, April 13, 2008; accepted April 24, 2008.

This work was presented as an extended research presentation at NAPCRG on October 21, 2007 in Vancouver, British Columbia.

Funding support: This study was funded by grant 1 P30AG021677 from the National Institute on Aging, grant D55HP05150 from the 
Health Resources and Services Administration, and a grant from the Robert Wood Johnson Foundation.

\section{References}

1. Cowie CC, Rust KF, Byrd-Holt DD, et al. Prevalence of diabetes and impaired fasting glucose in adults in the U.S. population: National Health And Nutrition Examination Survey 1999-2002. Diabetes Care. 2006;29(6):1263-1268.

2. Nichols GA, Hillier TA, Brown JB. Progression from newly acquired impaired fasting glusose to type 2 diabetes. Diabetes Care. 2007; $30(2): 228-233$

3. Brown JS, Vittinghoff $E$, Lin $F$, et al. Prevalence and risk factors for urinary incontinence in women with type 2 diabetes and impaired fasting glucose: findings from the National Health and Nutrition Examination Survey (NHANES) 2001-2002. Diabetes Care. 2006;29(6):1307-1312.

4. Nielson C, Lange T, Hadjokas N. Blood glucose and coronary artery disease in nondiabetic patients. Diabetes Care. 2006;29(5):998-1001.

5. Vehkavaara S, Seppala-Lindroos A, Westerbacka J, Groop PH, YkiJarvinen $\mathrm{H}$. In vivo endothelial dysfunction characterizes patients with impaired fasting glucose. Diabetes Care. 1999;22(12):2055-2060

6 . The cost-effectiveness of screening for type 2 diabetes. CDC Diabetes Cost-Effectiveness Study Group, Centers for Disease Control and Prevention. JAMA. 1998;280(20):1757-1763.

7. U.S. Preventive Services Task Force. Screening for type 2 diabetes mellitus in adults: recommendations and rationale. Ann Intern Med. 2003;138(3):212-214.

8. Harris R, Donahue K, Rathore SS, et al. Screening adults for type 2 diabetes: a review of the evidence for the U.S. Preventive Services Task Force. Ann Intern Med. 2003;138(3):215-229.

9. Zhang P, Engelgau MM, Valdez R, et al. Costs of screening for pre-diabetes among US adults: a comparison of different screening strategies. Diabetes Care. 2003;26(9):2536-2542.

10. DREAM (Diabetes REduction Assessment with ramipril and rosiglitazone Medication) Trial Investigators, Gerstein HC, Yusuf S, et al. Effect of rosiglitazone on the frequency of diabetes in patients with impaired glucose tolerance or impaired fasting glucose: a randomised controlled trial. Lancet. 2006;368(9541):1096-1105.

11. Haffner SM, Stern MP, Hazuda HP, Mitchell BD, Patterson JK. Cardiovascular risk factors in confirmed prediabetic individuals. Does the clock for coronary heart disease start ticking before the onset of clinical diabetes? JAMA. 1990;263(21):2893-2898.

12. Hanley AJ, Williams K, Stern MP, Haffner SM. Homeostasis mode assessment of insulin resistance in relation to the incidence of cardiovascular disease: the San Antonio Heart Study. Diabetes Care. 2002;25(7):1177-1184.

13. Knowler WC, Barrett-Connor E, Fowler SE, et al. Reduction in the incidence of type 2 diabetes with lifestyle intervention or metformin. N Engl J Med. 2002;346(6):393-403.

14. Nathan DM, Lachin J, Cleary $P$, et al. Intensive diabetes therapy and carotid intima-media thickness in type 1 diabetes mellitus. $N$ Engl J Med. 2003;348(23):2294-2303.

15. Pan XR, Li GW, Hu YH, et al. Effects of diet and exercise in preventing NIDDM in people with impaired glucose tolerance. The Da Qing IGT and Diabetes Study. Diabetes Care. 1997;20(4):537-544.

16. Tuomilehto J, Lindstrom J, Eriksson JG, et al. Prevention of type 2 diabetes mellitus by changes in lifestyle among subjects with impaired glucose tolerance. N Engl J Med. 2001;344(18):1343-1350.

17. UK Prospective Diabetes Study Group. Tight blood pressure control and risk of macrovascular and microvascular complications in type 2 diabetes: UKPDS 38. BMJ. 1998;317(7160):703-713.
18. Standards of medical care in diabetes-2008. Diabetes Care. 2008; 31(Suppl 1):S12-S54.

19. Glümer C, Carstensen B, Sandbæk A, et al. A Danish diabetes risk score for targeted screening: the Inter99 study. Diabetes Care. 2004;27(3):727-733.

20. Franciosi M, De Berardis G, Rossi MC, et al. Use of the diabetes risk score for opportunistic screening of undiagnosed diabetes and impaired glucose tolerance: the IGLOO (Impaired Glucose Tolerance and Long-Term Outcomes Observational) study. Diabetes Care. 2005;28(5):1187-1194.

21. Thomas C, Hyppönen E, Power C. Type 2 diabetes mellitus in midlife estimated from the Cambridge Risk Score and body mass index. Arch Intern Med. 2006;166(6):682-688.

22. Koopman RJ, Mainous AG, III, Diaz VA, Geesey ME. Changes in age at diagnosis of type 2 diabetes mellitus in the United States, 1988 to 2000. Ann Fam Med. 2005;3(1):60-63.

23. National Center for Health Statistics. The National Health and Nutrition Examination Survey (NHANES) analytic guidelines. Hyattsville, MD: US Department of Health and Human Services, Centers for Disease Control and Prevention, National Center for Health Statistics; 2004 [updated 2004 Jun; cited 2007 Apr 9]. http://www.cdc. gov/nchs/data/nhanes/nhanes_general_guidelines_june_04.pdf.

24. Mainous AG III, Koopman RJ, Diaz VA, et al. A coronary heart disease risk score based on patient-reported information. Am J Cardiol. 2007;99(9):1236-1241.

25. Pickering TG, Hall JE, Appel LJ, et al. Recommendations for blood pressure measurement in humans and experimental animals: part 1: blood pressure measurement in humans: a statement for professionals from the Subcommittee of Professional and Public Education of the American Heart Association Council on High Blood Pressure Research. Hypertension. 2005;45(1):142-161.

26. Ridker PM, Buring JE, Rifai N, Cook NR. Development and validation of improved algorithms for the assessment of global cardiovascular risk in women: the Reynolds Risk Score. JAMA. 2007;297(6):611-619.

27. Charlson ME, Pompei P, Ales KL, MacKenzie CR. A new method of classifying prognostic comorbidity in longitudinal studies: development and validation. J Chronic Dis. 1987;40(5):373-383.

28. MedCalc for Windows, Version 8.1. Mariakerke, Belgium: MedCalc Software; 2005.

29. Obuchowski NA, Lieber ML, Wians FH Jr. ROC curves in clinical chemistry: uses, misuses, and possible solutions. Clin Chem. 2004;50(7):1118-1125.

30. Pepe MS, Janes $H$, Longton $G$, Leisenring W, Newcomb P. Limitations of the odds ratio in gauging the performance of a diagnostic, prognostic, or screening marker. Am J Epidemiol. 2004;159(9):882-890.

31. Grover SA, Lowensteyn I, Joseph L, et al. Patient knowledge of coronary risk profile improves the effectiveness of dyslipidemia therapy: the CHECK-UP study: a randomized controlled trial. Arch Intern Med. 2007;167(21):2296-2303.

32. Lindstrom J, Tuomilehto J. The diabetes risk score: a practical too to predict type 2 diabetes risk. Diabetes Care. 2003;26(3):725-731

33. Schmidt MI, Duncan BB, Vigo A, et al. Detection of undiagnosed diabetes and other hyperglycemia states: the Atherosclerosis Risk in Communities Study. Diabetes Care. 2003;26(5):1338-1343.

34. Parikh NI, Pencina MJ, Wang TJ, et al. A risk score for predicting near-term incidence of hypertension: the Framingham Heart Study. Ann Intern Med. 2008;148(2):102-110.

35. Wilson PW, D'Agostino RB, Levy D, et al. Prediction of coronary heart disease using risk factor categories. Circulation. 1998; 97(18):1837-1847. 